\title{
Herbal Medicine for Adult Patients with Cough Variant Asthma: A Systematic Review and Meta-Analysis
}

\author{
Yuan-Bin Chen $\mathbb{D},{ }^{1}$ Johannah L Shergis $\mathbb{D},{ }^{2}$ Zhen-Hu Wu $\mathbb{D},{ }^{1}$ Xin-Feng Guo $\mathbb{D},{ }^{1}$ \\ Anthony L Zhang $\mathbb{D}^{2}{ }^{2}$ Lei Wu $\mathbb{D}^{1},{ }^{1}$ Fei-Ting Fan $\mathbb{D}^{1},{ }^{1}$ Yin-Ji Xu $\mathbb{D}^{1},{ }^{1}$ Charlie C Xue $\mathbb{D}^{1,2}$ \\ and Lin Lin ${ }^{1}$ \\ ${ }^{1}$ Department of Respiratory Medicine, The Second Clinical College of Guangzhou University of Chinese Medicine, \\ The Second Affiliated Hospital of Guangzhou University of Chinese Medicine, \\ Guangdong Provincial Hospital of Chinese Medicine, Guangzhou, China \\ ${ }^{2}$ School of Health and Biomedical Sciences, RMIT University, Bundoora, Victoria, Australia \\ Correspondence should be addressed to Charlie C Xue; charlie.xue@rmit.edu.au and Lin Lin; drlinlin620@163.com
}

Received 7 July 2020; Revised 9 February 2021; Accepted 22 February 2021; Published 2 March 2021

Academic Editor: Samra Bashir

Copyright $\odot 2021$ Yuan-Bin Chen et al. This is an open access article distributed under the Creative Commons Attribution License, which permits unrestricted use, distribution, and reproduction in any medium, provided the original work is properly cited.

\begin{abstract}
Introduction. Herbal medicine is commonly used by patients with chronic cough, but the role of herbal medicine for cough variant asthma (CVA) has not yet been clearly defined. For the first time, we performed a meta-analysis to integrate the current evidence of randomized controlled trials (RCTs) on this topic and assess the efficacy of herbal medicine in adults with CVA. Methods. A comprehensive search was conducted in electronic databases to identify RCTs of herbal medicine for adult CVA. Cochrane systematic review methods were followed, and the Grading of Recommendations Assessment, Development, and Evaluation was performed to evaluate the quality of evidence. Results. Twenty-eight RCTs were included. Compared with placebo, moderatequality evidence from two studies showed that herbal medicine was associated with reduced cough symptom score (CSS) (MD -1.15 points; $95 \% \mathrm{CI},-1.67$ to -0.63 ) and visual analogue scale (VAS) (MD -1.76 points; $95 \% \mathrm{CI},-2.66$ to -0.86 ). Compared with montelukast, low- to moderate-quality evidence from 11 studies indicated that herbal medicine was associated with improved Leicester Cough Questionnaire (LCQ) (MD 2.38 points; 95\% CI, 1.32 to 3.44), reduced CSS (SMD -0.81 points; $95 \%$ CI, -1.09 to -0.53 ), and VAS (MD -1.34 points; $95 \% \mathrm{CI},-1.82$ to -0.86 ). There were no significant differences between herbal medicine and ICS plus bronchodilator. Conclusions. In adults with CVA, herbal medicine may result in improved quality of life and reduced cough frequency and severity scores compared with placebo or montelukast. Herbal medicine was not better than ICS plus a bronchodilator but the evidence is very uncertain.
\end{abstract}

\section{Introduction}

Cough variant asthma (CVA) is a form of asthma with bronchial hyperresponsiveness eosinophilic airway inflammation and airway remodeling. It presents solely with a dry or minimally productive cough, without the traditional asthma symptoms of wheezing and shortness of breath [1]. The three most common types of chronic cough, accounting for approximately $90 \%$ of cases, include CVA, upper airway cough syndrome, and gastroesophageal reflux disease [2,3]. Moreover, patients in Asian countries experience CVA more commonly than other types of chronic cough $[4,5]$.
Cough variant asthma treatment is similar to typical asthma. First-line treatments should include inhaled corticosteroids (ICS) and long-acting beta-agonists, avoiding triggers [6]. If these treatments are not successful, leukotriene receptor antagonists or oral corticosteroids may be used but have limited efficacy [7]. However, the standardized treatment of CVA is limited by the following: (1) the lack of inhaled therapeutic drugs such as Advair Diskus ${ }^{\circledR}$ in community and grassroots hospitals in developing countries; (2) adverse reactions caused by ICS, for example, hoarseness, mouth ulcers, and pneumonia [8, 9]; (3) patients insensitivity or intolerance to corticosteroid therapy; and (4) 
people being cautious of using corticosteroids. Therefore, it is not uncommon that patients with CVA do not receive a standardized treatment plan at the beginning or are unable to adhere to the treatment, especially in China.

Although it is acknowledged that there is a lack of highquality evidence on herbal medicine for CVA, broad-based population surveys indicate that a growing number of patients are using herbal medicine for a range of chronic diseases $[10,11]$. Cough is the most common complaint when patients seek medical treatment [12], and herbal medicine may be one of the important options for many patients. In recent years, more and more clinical studies on herbal medicine for CVA have emerged. According to current Chinese national guidelines of cough, herbal medicine is considered to be effective and Suhuang Zhike capsules are recommended for CVA [13]. However, due to the absence of evidence from systematic reviews, clinicians' confidence in herbal medicine for CVA is low, and the specific role(s) of herbal medicine in the treatment of CVA is unclear. The aim of this systematic review and meta-analysis was, therefore, to evaluate the efficacy and safety of herbal medicine in adult patients with CVA.

\section{Methods}

This meta-analysis was performed using Review Manager according to the Cochrane Handbook for Systematic Reviews of Interventions (Version 5.3.3) [14] and followed the statement based on the Preferred Reporting Items for Systematic Reviews and Meta-analyses guidelines [15]. The protocol for this review was registered in PROSPERO (Identifier: CRD42018115083).

2.1. Search Strategy. Evidence was gathered by searching electronic English and Chinese language databases and the methods followed the Cochrane Handbook of Systematic Reviews [14]. English language databases included PubMed, Cochrane Library, Excerpta Medica Database (Embase), Cumulative Index of Nursing and Allied Health Literature, Cochrane Central Register of Controlled Trials, and Allied and Complementary Medicine Database. Chinese language databases included China BioMedical Literature, China National Knowledge Infrastructure, Chonqing VIP, and Wanfang. Databases were searched from their inception to April 2020 without language restrictions. Our search strategy was the combination of subject words and keywords including herbal medicine, CVA, and their synonyms. We also scanned reference lists of the included studies and contacted authors to acquire unpublished data.

2.2. Eligibility Criteria. Trials were selected based on the following inclusion criteria: (1) participants with CVA according to the American Practice Guidelines [12] or Diagnosis and Chinese Cough Guidelines [13]; (2) randomized controlled trials (RCTs) comparing herbal medicine with placebo, leukotriene receptor antagonists, or ICS plus bronchodilators; (3) trials recruiting adults older than 18 years; (4) treatment duration at least two weeks; and (5) endpoints meeting any of our prespecified outcomes of interest. Exclusion criteria were (1) trials of participants with typical asthma, (2) trials including children with CVA, and (3) trials using different pharmacotherapy cointerventions in the intervention and control groups.

2.3. Data Extraction. Two reviewers (YBC, JLS) independently extracted the following details from each study using EpiData software: title, authors, publication year, country of origin, patient characteristics, study design, doses and duration of intervention measures, study outcomes, and adverse events. Disagreements were resolved by discussion and consultation with a third person (ALZ). The primary outcomes were Leicester Cough Questionnaire (LCQ) [16], cough symptom score (CSS) [17, 18], and visual analogue scale (VAS). The secondary outcomes included the forced expiratory volume in one second $\left(\mathrm{FEV}_{1}\right), \mathrm{CD} 4+/ \mathrm{CD} 8+$ ratio, effective rate $[19,20]$, and adverse events.

2.4. Risk of Bias Assessment. The risk of bias was independently evaluated according to the Cochrane Collaboration's Risk of Bias Tool [14]. Bias in each trial can be categorized into seven items including the randomization sequence generation, allocation concealment, blinding of participants and personnel, blinding of outcome assessment, incomplete outcome data, selective reporting, and other bias. Each domain was assessed to determine whether bias is at low, high, or unclear risk.

2.5. Statistical Analysis. We used a random-effects model to pool the available data. Estimates of heterogeneity were reported with risk ratio (RR), mean difference (MD) or standardized mean difference (SMD), and 95\% confidence intervals (CIs). Formal tests for heterogeneity between summary data were performed using the $I^{2}$ statistic. An $I^{2}>$ $50 \%$ was taken to indicate substantial heterogeneity. The sensitivity analysis was undertaken to explore potential sources of heterogeneity, based on the low risk of bias for the risk of bias domain sequence generation or studies with characteristics different from the others. Where possible and appropriate, planned subgroup analysis included duration of treatment and specific interventions to determine whether the subgroups significantly differed from one another. The statistical analysis was conducted with Review Manager version 5.3.3 software.

2.6. Quality of Evidence. An assessment of the strength and quality (certainty) of the evidence from RCTs was made using Grading of Recommendations Assessment, Development, and Evaluation (GRADE) [21]. The certainty of the evidence for each outcome was rated according to the outline in the GRADE approach.

\section{Results}

3.1. Studies Retrieved and Characteristics. The process of study selection is shown in Figure 1. Twenty-eight RCTs 


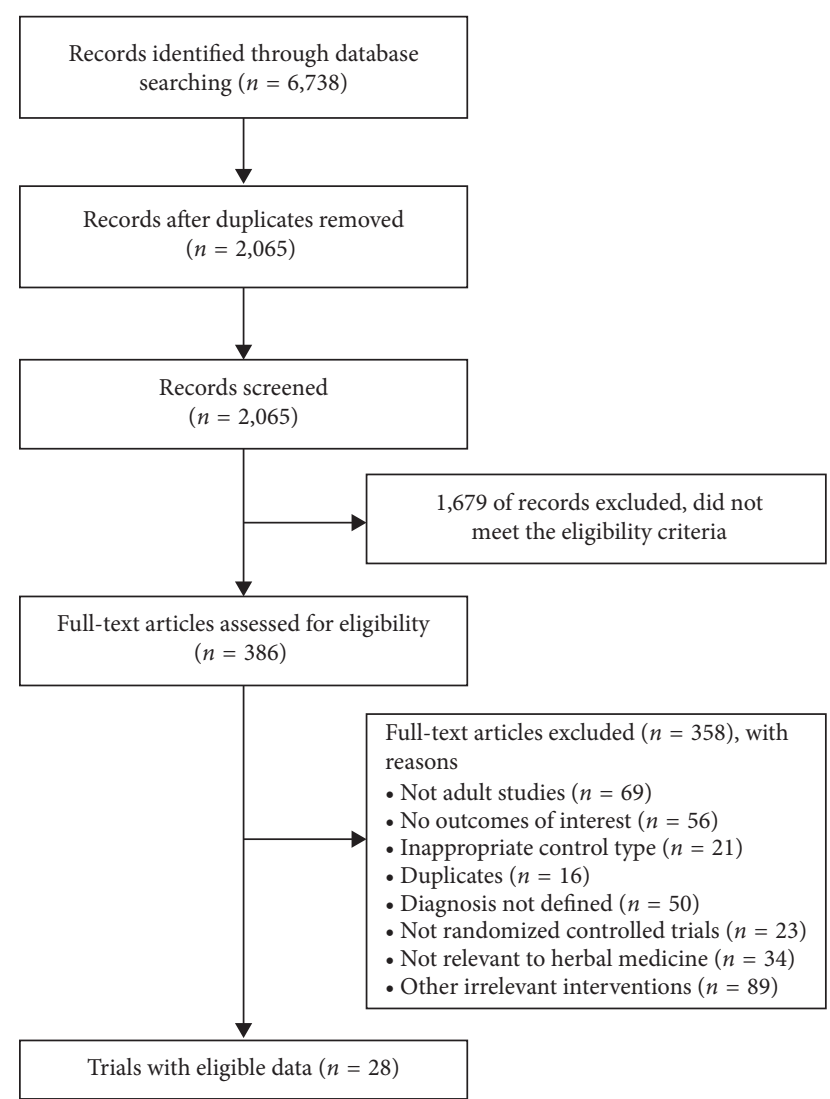

FIGURE 1: Flow chart of the study selection process.

including 2,079 patients with CVA fulfilled the eligibility criteria [22-49]. All studies included two intervention arms except for four studies that included three arms; data from the two relevant arms were included in the analysis [38, 45, 48, 49].

Characteristics of the studies are summarized in Table 1 . The 28 studies were divided into three groups for analysis: (1) herbal medicine versus placebo, (2) herbal medicine versus leukotriene receptor antagonists, and (3) herbal medicine versus ICS plus bronchodilator. Results for each grouping are presented in the GRADE summary of findings tables (Tables S1-S3) and metaanalysis forest plots (Figures 2-5 and Figures S2-S5).

Chinese medicine syndrome classification was used in 19 studies [23-25, 27, 29, 30, 32-35, 37, 39, 42-48]. The most frequently reported syndrome was severe wind attacking the lungs (8 studies) $[23,25,30,32,35,45-47]$. All formulae of herbal medicine were administered orally, with decoction, granule powder, or capsules. In 11 studies [24-34], montelukast $10 \mathrm{mg}$ was given as a comparator. In the studies that used ICS plus bronchodilator as a comparator, salmeterol and fluticasone propionate were used in 10 studies [35, 37, 39-42, 44-46, 48], budesonide and formoterol fumarate in four studies $[38,43,47,49]$, and budesonide and salbutamol in one study [36]. The duration of treatment ranged from 2 to 12 weeks.

3.2. Risk of Bias. All studies specified that "randomization" was used in the allocation of participants to the herbal medicine intervention or the control groups. However, $42.9 \%$ of studies $(n=12)$ did not clearly specify the method of random sequence generation $[27,28,30,31,35,38,39,43$, $44,46,47,49]$. Three studies described an adequate method of allocation concealment and were assessed as low risk of bias $[22,23,37]$. Two studies were evaluated as low risk of bias in the blinding of participants and personnel because a placebo of herbal medicine was used as the comparator [22, 23]. Other studies lacked information about blinding of participants and personnel and were at high risk of bias. Two studies specified the information of blinding of outcome assessors [22, 23] and were assessed as low risk. The majority $(89.3 \%)$ were at low risk because there was no missing data or dropouts were balanced between groups [22-41, 43-48]. One study did not report its predefined primary outcome without reason and was at high risk of reporting bias. The risk of bias is summarized in Figure S1.

\subsection{Results of Herbal Medicine versus Montelukast}

3.3.1. Leicester Cough Questionnaire (LCQ). Low-quality evidence from six studies used the LCQ to assess health-related quality of life in 422 participants (Table S1) $[24,25,27,29,33,34]$. The result indicated that participants receiving herbal medicine had significantly increased LCQ-total scores (indicating reduced symptoms) than those receiving montelukast, and the difference achieved the minimal clinically important difference of 1.3 points [50], although the heterogeneity was substantial (MD 2.38 points; $95 \% \mathrm{CI}, 1.32$ to 3.44 ; $p<0.00001, I^{2}=84 \%$ ) (Figure 2).

The LCQ that comprises three domains (physical, psychological, and social) was available in five studies. The physical score increased in the herbal medicine group more than the montelukast group, heterogeneity might not be important (MD 0.74 points; 95\% CI, 0.44 to 1.03; $p<0.00001, I^{2}=40 \%$ ), and social score also improved (MD 0.49 points; $95 \% \mathrm{CI}, 0.14$ to $\left.0.85 ; p=0.0006, I^{2}=60 \%\right)$. However, there was no difference between groups in terms of psychological score (MD 0.34 points; $95 \% \mathrm{CI},-0.17$ to $0.84 ; p=0.19, I^{2}=84 \%$ ) (Figure 2 ).

3.3.2. Cough Symptom Score (CSS). Low-quality evidence from six studies (454 participants) evaluated CSS (Table S1) $[24,26,28,31-33]$. The pooled result indicated that herbal medicine was superior to montelukast, and the heterogeneity was moderate (SMD -0.80 points; $95 \% \mathrm{CI},-1.08$ to $-0.51 ; p<0.00001, I^{2}=53 \%$ ) (Figure 3 ).

3.3.3. Visual Analogue Scale (VAS). Low-quality evidence from one RCT with 77 participants assessed cough VAS using a numerical rating scale (Table S1) [34]. The cough was reduced in participants receiving herbal medicine compared to those receiving montelukast ( $\mathrm{MD}-1.34$ points; $95 \% \mathrm{CI}$, -1.82 to -0.86 ) (Figure 4 ).

3.3.4. Forced Expiratory Volume in One Second (FEV1). Low-quality evidence from two studies (148 participants) assessed $\mathrm{FEV}_{1}$ (Table $\mathrm{S} 1$ ). One study reported $\mathrm{FEV}_{1}$ liters $(L)$ 
TABLE 1: Characteristics of the included studies.

\begin{tabular}{|c|c|c|c|c|c|c|c|c|}
\hline $\begin{array}{l}\text { Author } \\
\text { (year) }\end{array}$ & Study design & $\begin{array}{l}\text { Number of } \\
\text { participants } \\
\text { (I/C) }\end{array}$ & $\begin{array}{l}\text { Age, years } \\
\text { (mean, } \\
\text { standard } \\
\text { deviation) }\end{array}$ & $\begin{array}{c}\text { Gender } \\
(\mathrm{M} / \mathrm{F}) \\
(\%)\end{array}$ & $\begin{array}{c}\text { Intervention/ } \\
\text { dose/frequency }\end{array}$ & $\begin{array}{l}\text { Control/dose/ } \\
\text { frequency }\end{array}$ & $\begin{array}{c}\text { Treatment } \\
\text { duration } \\
\text { (weeks) }\end{array}$ & Outcomes * \\
\hline $\begin{array}{l}\text { Zhang } \\
\text { et al. } \\
(2009) \\
{[23]}\end{array}$ & $\begin{array}{l}\text { Randomized, } \\
\text { double-blind, } \\
\text { placebo- } \\
\text { controlled }\end{array}$ & $74(36 / 38)$ & $\begin{array}{c}\text { I: } 33.42 \pm 11.26 \\
\text { C: } \\
34.35 \pm 10.90\end{array}$ & $51.4 / 48.6$ & $\begin{array}{l}\text { Yu-ping-feng } \\
\text { san, twice a day }\end{array}$ & $\begin{array}{c}\text { Placebo, twice a } \\
\text { day }\end{array}$ & 8 & (5) 7 \\
\hline $\begin{array}{l}\text { Wang } \\
\text { et al. } \\
(2017) \\
{[24]}\end{array}$ & $\begin{array}{c}\text { Randomized, } \\
\text { double-blind, } \\
\text { placebo- } \\
\text { controlled }\end{array}$ & $80(40 / 40)$ & $18-75$ & $29.2 / 70.8$ & $\begin{array}{l}\text { Qu-feng-xuan-fei } \\
\text { formula, one } \\
\text { dose a day }\end{array}$ & $\begin{array}{l}\text { Placebo, one } \\
\text { dose a day }\end{array}$ & 2 & (2) (3) \\
\hline $\begin{array}{l}\text { Miao } \\
\text { et al. } \\
(2012) \\
{[25]}\end{array}$ & $\begin{array}{l}\text { Randomized, } \\
\text { open-label trial }\end{array}$ & $84(56 / 28)$ & $\begin{array}{c}\text { I: } 45.81 \pm 13.31 \\
\text { C: } \\
43.85 \pm 11.45\end{array}$ & $31.0 / 69.0$ & $\begin{array}{c}\text { Self-designed } \\
\text { formula, one } \\
\text { dose a day }\end{array}$ & $\begin{array}{c}\text { Montelukast } \\
10 \mathrm{mg} \text {, once daily }\end{array}$ & 4 & (1) (2) (6) \\
\hline $\begin{array}{l}\text { Cong } \\
\text { et al. } \\
(2013) \\
{[26]}\end{array}$ & $\begin{array}{l}\text { Randomized, } \\
\text { open-label trial }\end{array}$ & $60(30 / 30)$ & $\begin{array}{c}\text { I: } 47.41 \pm 13.34 \\
\text { C: } \\
45.57 \pm 13.34\end{array}$ & $35.1 / 64.9$ & $\begin{array}{l}\text { Wen-run-xin-jin } \\
\text { fang, one dose a } \\
\text { day }\end{array}$ & $\begin{array}{c}\text { Montelukast } \\
10 \mathrm{mg} \text {, once daily }\end{array}$ & 2 & (1) (6) \\
\hline $\begin{array}{l}\text { Zhang } \\
\text { et al. } \\
(2014) \\
{[27]}\end{array}$ & $\begin{array}{l}\text { Randomized, } \\
\text { open-label trial }\end{array}$ & $48(24 / 24)$ & $\begin{array}{c}\text { I: } 41.17 \pm 13.69 \\
\text { C: } \\
41.25 \pm 14.01\end{array}$ & $37.5 / 62.5$ & $\begin{array}{l}\text { Hua-gai san, one } \\
\text { dose a day }\end{array}$ & $\begin{array}{c}\text { Montelukast } \\
10 \mathrm{mg} \text {, once daily }\end{array}$ & 2 & (2) (4) (6) (7) \\
\hline $\begin{array}{l}\text { Ye et al. } \\
(2015) \\
{[28]}\end{array}$ & $\begin{array}{l}\text { Randomized, } \\
\text { open-label trial }\end{array}$ & $60(30 / 30)$ & $\begin{array}{l}\text { I: } 43.37 \pm 3.34 \\
\text { C: } 44.00 \pm 3.37\end{array}$ & $40.0 / 60.0$ & $\begin{array}{c}\text { Wen-dan } \\
\text { formula, once } \\
\text { daily }\end{array}$ & $\begin{array}{c}\text { Montelukast } \\
10 \mathrm{mg} \text {, once daily }\end{array}$ & 4 & (1) \\
\hline $\begin{array}{l}\text { Wu et al. } \\
(2016) \\
{[29]}\end{array}$ & $\begin{array}{l}\text { Randomized, } \\
\text { open-label trial }\end{array}$ & $80(40 / 40)$ & $\begin{array}{l}\text { I: } 43.63 \pm 8.74 \\
\text { C: } 39.52 \pm 9.16\end{array}$ & $52.5 / 47.5$ & $\begin{array}{l}\text { Bu-zhong-yi-qi } \\
\text { formula, one } \\
\text { dose a day }\end{array}$ & $\begin{array}{c}\text { Montelukast } \\
10 \mathrm{mg} \text {, once daily }\end{array}$ & 8 & (2) (6) (7) \\
\hline $\begin{array}{l}\text { Yu et al. } \\
(2016) \\
{[30]}\end{array}$ & $\begin{array}{l}\text { Randomized, } \\
\text { open-label trial }\end{array}$ & $60(30 / 30)$ & $\begin{array}{c}\text { I: } 35.30 \pm 10.77 \\
\text { C: } \\
37.93 \pm 12.69\end{array}$ & $41.7 / 58.3$ & $\begin{array}{l}\text { Qu-feng-xuan-fei } \\
\text { formula, one } \\
\text { dose a day }\end{array}$ & $\begin{array}{c}\text { Montelukast } \\
10 \mathrm{mg} \text {, once daily }\end{array}$ & 4 & (1) (6) (7) \\
\hline $\begin{array}{l}\text { Kang } \\
\text { et al. } \\
(2017) \\
{[31]}\end{array}$ & $\begin{array}{l}\text { Randomized, } \\
\text { open-label trial }\end{array}$ & $100(50 / 50)$ & $\begin{array}{l}\text { I: } 35.60 \pm 6.20 \\
\text { C: } 34.70 \pm 7.80\end{array}$ & $54.0 / 46.0$ & $\begin{array}{l}\text { Chan-yi-he-ji, } \\
\text { one dose a day }\end{array}$ & $\begin{array}{c}\text { Montelukast } \\
10 \mathrm{mg} \text {, once daily }\end{array}$ & 4 & (4) (6) \\
\hline $\begin{array}{l}\text { Sun et al. } \\
(2017) \\
{[32]}\end{array}$ & $\begin{array}{l}\text { Randomized, } \\
\text { open-label trial }\end{array}$ & $98(49 / 49)$ & $\begin{array}{l}\text { I: } 45.51 \pm 5.36 \\
\text { C: } 47.61 \pm 5.62\end{array}$ & $51.0 / 49.0$ & $\begin{array}{c}\text { Su-huang-zhi-ke } \\
\text { capsules, } 3 \\
\text { capsules, } 3 \text { times } \\
\text { a day }\end{array}$ & $\begin{array}{c}\text { Montelukast } \\
10 \mathrm{mg} \text {, once daily }\end{array}$ & 2 & (2) (6) (7) \\
\hline $\begin{array}{l}\text { Teng } \\
\text { et al. } \\
(2017) \\
{[33]}\end{array}$ & $\begin{array}{l}\text { Randomized, } \\
\text { open-label trial }\end{array}$ & $60(30 / 30)$ & $\begin{array}{l}\text { I: } 53.77 \pm 6.63 \\
\text { C: } 52.87 \pm 4.89\end{array}$ & $38.3 / 61.7$ & $\begin{array}{l}\text { Zhi-ke-ping- } \\
\text { chuan formula, } \\
\text { one dose a day }\end{array}$ & $\begin{array}{c}\text { Montelukast } \\
10 \mathrm{mg} \text {, once daily }\end{array}$ & 2 & (2) (6) \\
\hline $\begin{array}{l}\text { Yan et al. } \\
(2017) \\
{[34]}\end{array}$ & $\begin{array}{l}\text { Randomized, } \\
\text { open-label trial }\end{array}$ & $90(45 / 45)$ & $\begin{array}{l}\text { I: } 35.62 \pm 6.55 \\
\text { C: } 35.51 \pm 6.65\end{array}$ & $48.9 / 51.1$ & $\begin{array}{l}\text { Yi-qi-qu-feng } \\
\text { formula, one } \\
\text { dose a day }\end{array}$ & $\begin{array}{c}\text { Montelukast } \\
10 \mathrm{mg} \text {, once daily }\end{array}$ & 4 & (1) (2) (6) \\
\hline $\begin{array}{l}\text { Cao et al. } \\
(2018) \\
{[35]}\end{array}$ & $\begin{array}{l}\text { Randomized, } \\
\text { open-label trial }\end{array}$ & $80(40 / 40)$ & $\begin{array}{l}\text { I: } 69.68 \pm 4.54 \\
\text { C: } 71.03 \pm 4.67\end{array}$ & $54.6 / 45.4$ & $\begin{array}{l}\text { Jia-wei-ma-xing- } \\
\text { er-chen formula, } \\
\text { one dose a day }\end{array}$ & $\begin{array}{c}\text { Montelukast } \\
10 \mathrm{mg} \text {, once daily }\end{array}$ & 4 & (1) (3) (6) (7) \\
\hline $\begin{array}{l}\text { Luo et al. } \\
(2009) \\
{[36]}\end{array}$ & $\begin{array}{l}\text { Randomized, } \\
\text { open-label trial }\end{array}$ & $60(30 / 30)$ & $\begin{array}{l}\text { I: } 41.97 \pm 10.97 \\
\text { C: } 43.43 \pm 9.51\end{array}$ & $55.0 / 45.0$ & $\begin{array}{l}\text { Ke-ping } \\
\text { formula, one } \\
\text { dose a day }\end{array}$ & $\begin{array}{c}\text { Seretide } 50 / \\
100 \mu \mathrm{g} \text {, twice a } \\
\text { day }\end{array}$ & 4 & (6) (7) \\
\hline $\begin{array}{l}\text { Zhang } \\
\text { et al. } \\
(2013) \\
{[37]}\end{array}$ & $\begin{array}{l}\text { Randomized, } \\
\text { open-label trial }\end{array}$ & $140(70 / 70)$ & $\begin{array}{c}\text { I: } 37.40 \pm 11.20 \\
\text { C: } \\
38.10 \pm 10.50\end{array}$ & $39.8 / 60.2$ & $\begin{array}{l}\text { Gu-ben-zhi-ke } \\
\text { granule, one } \\
\text { dose a day }\end{array}$ & $\begin{array}{c}\text { Budesonide } \\
0.2 \mathrm{mg} \text { plus } \\
\text { salbutamol } \\
200 \mu \mathrm{g} \text {, twice a } \\
\text { day }\end{array}$ & 8 & (2) (7) \\
\hline
\end{tabular}


TABLE 1: Continued.

\begin{tabular}{|c|c|c|c|c|c|c|c|c|}
\hline $\begin{array}{l}\text { Author } \\
\text { (year) }\end{array}$ & Study design & $\begin{array}{l}\text { Number of } \\
\text { participants } \\
\text { (I/C) }\end{array}$ & $\begin{array}{l}\text { Age, years } \\
\text { (mean, } \\
\text { standard } \\
\text { deviation) }\end{array}$ & $\begin{array}{c}\text { Gender } \\
(\mathrm{M} / \mathrm{F}) \\
(\%)\end{array}$ & $\begin{array}{c}\text { Intervention/ } \\
\text { dose/frequency }\end{array}$ & $\begin{array}{l}\text { Control/dose/ } \\
\text { frequency }\end{array}$ & $\begin{array}{c}\text { Treatment } \\
\text { duration } \\
\text { (weeks) }\end{array}$ & Outcomes* \\
\hline $\begin{array}{l}\text { Wang } \\
\text { et al. } \\
(2014) \\
{[38]}\end{array}$ & $\begin{array}{l}\text { Randomized, } \\
\text { open-label trial }\end{array}$ & $62(31 / 31)$ & $\begin{array}{c}\text { I: } 45.71 \pm 12.28 \\
\text { C: } \\
44.43 \pm 11.61\end{array}$ & $42.6 / 57.4$ & $\begin{array}{c}\text { Wen-dan } \\
\text { formula, once } \\
\text { daily }\end{array}$ & $\begin{array}{c}\text { Seretide } 50 / \\
250 \mu \mathrm{g} \text {, twice a } \\
\text { day }\end{array}$ & 2 & (1) (7) \\
\hline $\begin{array}{l}\text { Zhuo } \\
\text { et al. } \\
(2014) \\
{[39]}\end{array}$ & $\begin{array}{l}\text { Randomized, } \\
\text { three-arms, } \\
\text { open-label trial }\end{array}$ & $93(31 / 29)$ & $\begin{array}{c}\text { I: } 35.10 \pm 10.90 \\
\text { C: } \\
38.20 \pm 11.00\end{array}$ & $57.0 / 43.0$ & $\begin{array}{c}\text { Su-huang-zhi-ke } \\
\text { capsules, } 3 \\
\text { capsules, } 3 \text { times } \\
\text { a day }\end{array}$ & $\begin{array}{c}\text { Symbicort } \\
\text { Turbuhaler } 160 / \\
4.5 \mu \mathrm{g} \text {, twice a } \\
\text { day }\end{array}$ & 4 & (2) (3) (7) \\
\hline $\begin{array}{l}\text { Xin et al. } \\
(2015) \\
{[40]}\end{array}$ & $\begin{array}{l}\text { Randomized, } \\
\text { open-label trial }\end{array}$ & $118(60 / 58)$ & $22-66$ & $53.4 / 46.6$ & $\begin{array}{l}\text { Jiu-xian san, } \\
\text { once daily }\end{array}$ & $\begin{array}{c}\text { Seretide } 50 / \\
250 \mu \mathrm{g}, \text { twice a } \\
\text { day }\end{array}$ & 2 & (4) (6) \\
\hline $\begin{array}{l}\text { Yang } \\
\text { et al. } \\
(2015) \\
{[41]}\end{array}$ & $\begin{array}{l}\text { Randomized, } \\
\text { open-label trial }\end{array}$ & $60(30 / 30)$ & $\begin{array}{c}\text { I: } 45.85 \pm 13.76 \\
\text { C: } \\
47.02 \pm 12.58\end{array}$ & $33.3 / 66.7$ & $\begin{array}{c}\text { Self-designed } \\
\text { formula, once } \\
\text { daily }\end{array}$ & $\begin{array}{c}\text { Seretide } 50 / \\
100 \mu \mathrm{g}, \text { twice a } \\
\text { day }\end{array}$ & 8 & (2) (6) \\
\hline $\begin{array}{l}\text { Zhao } \\
\text { et al. } \\
(2015) \\
{[42]}\end{array}$ & $\begin{array}{l}\text { Randomized, } \\
\text { open-label trial }\end{array}$ & $92(46 / 46)$ & $\begin{array}{c}\text { I: } 52.68 \pm 16.42 \\
\text { C: } \\
49.35 \pm 14.27\end{array}$ & $52.2 / 47.8$ & $\begin{array}{l}\text { Yi-qi-bu-shen } \\
\text { formula, once } \\
\text { daily }\end{array}$ & $\begin{array}{c}\text { Seretide } 50 / \\
250 \mu \mathrm{g} \text {, twice a } \\
\text { day }\end{array}$ & 8 & (4) (6) \\
\hline $\begin{array}{l}\text { Qin et al. } \\
(2016) \\
{[43]}\end{array}$ & $\begin{array}{l}\text { Randomized, } \\
\text { open-label trial }\end{array}$ & $63(30 / 33)$ & $\begin{array}{c}\text { I: } 39.23 \pm 12.41 \\
\text { C: } \\
38.63 \pm 13.87\end{array}$ & $41.3 / 58.7$ & $\begin{array}{l}\text { Chai-hu-zhi-ju } \\
\text { formula, once } \\
\text { daily }\end{array}$ & $\begin{array}{c}\text { Seretide } 50 / \\
250 \mu \mathrm{g} \text {, twice a } \\
\text { day }\end{array}$ & 2 & (3) (6) (7) \\
\hline $\begin{array}{l}\text { Sun et al. } \\
(2016) \\
{[44]}\end{array}$ & $\begin{array}{l}\text { Randomized, } \\
\text { open-label trial }\end{array}$ & $64(32 / 32)$ & $\begin{array}{c}\text { I: } 34.19 \pm 11.09 \\
\text { C: } \\
33.84 \pm 11.43\end{array}$ & $51.6 / 48.4$ & $\begin{array}{c}\text { Yang-yin-qu-feng } \\
\text { formula, once } \\
\text { daily }\end{array}$ & $\begin{array}{c}\text { Symbicort } \\
\text { Turbuhaler } 160 / \\
4.5 \mu \mathrm{g}, \text { twice a } \\
\text { day }\end{array}$ & 4 & (4) 7 \\
\hline $\begin{array}{l}\text { Zhang } \\
\text { et al. } \\
(2016) \\
{[45]}\end{array}$ & $\begin{array}{l}\text { Randomized, } \\
\text { open-label trial }\end{array}$ & $71(38 / 31)$ & $\begin{array}{l}\text { I: } 37.24 \pm 1.78 \\
\text { C: } 39.73 \pm 2.42\end{array}$ & $36.0 / 64.0$ & $\begin{array}{l}\text { Qu-feng-hua-tan } \\
\text { formula, once } \\
\text { daily }\end{array}$ & $\begin{array}{c}\text { Seretide } 50 / \\
250 \mu \mathrm{g} \text {, twice a } \\
\text { day }\end{array}$ & 4 & (3) \\
\hline $\begin{array}{l}\text { Lu et al. } \\
(2016) \\
{[46]}\end{array}$ & $\begin{array}{l}\text { Randomized, } \\
\text { three-arms,, } \\
\text { open-label trial }\end{array}$ & $120(40 / 40)$ & $\begin{array}{c}\text { I: } 46.23 \pm 12.59 \\
\text { C: } \\
44.00 \pm 13.79\end{array}$ & $30.8 / 69.2$ & $\begin{array}{c}\text { Shu-feng-zhi-sou } \\
\text { formula, once } \\
\text { daily }\end{array}$ & $\begin{array}{c}\text { Seretide } 50 / \\
250 \mu \mathrm{g} \text {, twice a } \\
\text { day }\end{array}$ & 4 & (4) (7) \\
\hline $\begin{array}{l}\text { Cui et al. } \\
(2017) \\
{[47]}\end{array}$ & $\begin{array}{l}\text { Randomized, } \\
\text { open-label trial }\end{array}$ & $60(30 / 30)$ & $\begin{array}{c}\text { I: } 45.63 \pm 13.67 \\
\text { C: } \\
47.21 \pm 14.42\end{array}$ & $48.3 / 51.7$ & $\begin{array}{l}\text { Bu-shen-qu- } \\
\text { feng-zhi-ke } \\
\text { formula, once } \\
\text { daily }\end{array}$ & $\begin{array}{c}\text { Seretide } 50 / \\
250 \mu \mathrm{g}, \text { twice a } \\
\text { day }\end{array}$ & 8 & (2) (6) (7) \\
\hline $\begin{array}{l}\text { Huang } \\
\text { et al. } \\
(2017) \\
{[48]}\end{array}$ & $\begin{array}{l}\text { Randomized, } \\
\text { open-label trial }\end{array}$ & $102(50 / 52)$ & $\begin{array}{l}\text { I: } 32.79 \pm 6.58 \\
\text { C: } 34.21 \pm 8.58\end{array}$ & $48.9 / 51.1$ & $\begin{array}{l}\text { Qu-feng-zhi-ke } \\
\text { formula, once } \\
\text { daily }\end{array}$ & $\begin{array}{c}\text { Symbicort } \\
\text { Turbuhaler } 80 / \\
4.5 \mu \mathrm{g} \text {, twice a } \\
\text { day }\end{array}$ & 2 & (3) (6) \\
\hline $\begin{array}{l}\text { Lu et al. } \\
(2017) \\
{[49]}\end{array}$ & $\begin{array}{l}\text { Randomized, } \\
\text { three-arms, } \\
\text { open-label trial }\end{array}$ & $90(30 / 30)$ & $\begin{array}{c}\text { I: } 42.70 \pm 13.35 \\
\text { C: } \\
43.98 \pm 11.70\end{array}$ & 28.9/71.1 & $\begin{array}{l}\text { Tiao-zhong-yi-fei } \\
\text { formula, once } \\
\text { daily }\end{array}$ & $\begin{array}{c}\text { Seretide } 50 / \\
250 \mu \mathrm{g}, \text { twice a } \\
\text { day }\end{array}$ & 12 & (4) \\
\hline $\begin{array}{l}\text { Qiu et al. } \\
\text { (2017) } \\
{[50]}\end{array}$ & $\begin{array}{c}\text { Randomized, } \\
\text { three-arms }\end{array}$ & $98(31 / 29)$ & $30.10 \pm 8.20$ & $49.0 / 51.0$ & $\begin{array}{c}\text { Su-huang-zhi-ke } \\
\text { capsules, } 3 \\
\text { capsules, } 3 \text { times } \\
\text { a day }\end{array}$ & $\begin{array}{c}\text { Symbicort } \\
\text { Turbuhaler } 160 / \\
4.5 \mu \mathrm{g}, \text { twice a } \\
\text { day }\end{array}$ & 4 & (2) \\
\hline
\end{tabular}

* Outcome measures included (1)Leicester Cough Questionnaire; (2)cough symptom score; (3)visual analogue scale; (4)forced expiratory volume in one second; (5)immune function; (6) effective rate; (7) adverse events.

[30], and one reported $\mathrm{FEV}_{1}$ percentage (\%) [26]. $\mathrm{FEV}_{1}(L)$ increased in participants receiving herbal medicine compared to montelukast (MD $0.29 \mathrm{~L} ; 95 \% \mathrm{CI}, 0.07$ to 0.51 ), and $\mathrm{FEV}_{1} \%$ also improved (MD 2.84\%; $95 \% \mathrm{CI}, 0.35$ to 5.33 ) (Figure S3).
3.3.5. Effective Rate. Moderate-quality evidence from 10 studies [24-26, 28-34], including 748 participants, assessed the effective rate (Table S1). The effective rate was classified as the number of people with improved symptoms. Treatments were classified as effective in people with clinically 
(1) LCQ-total scores

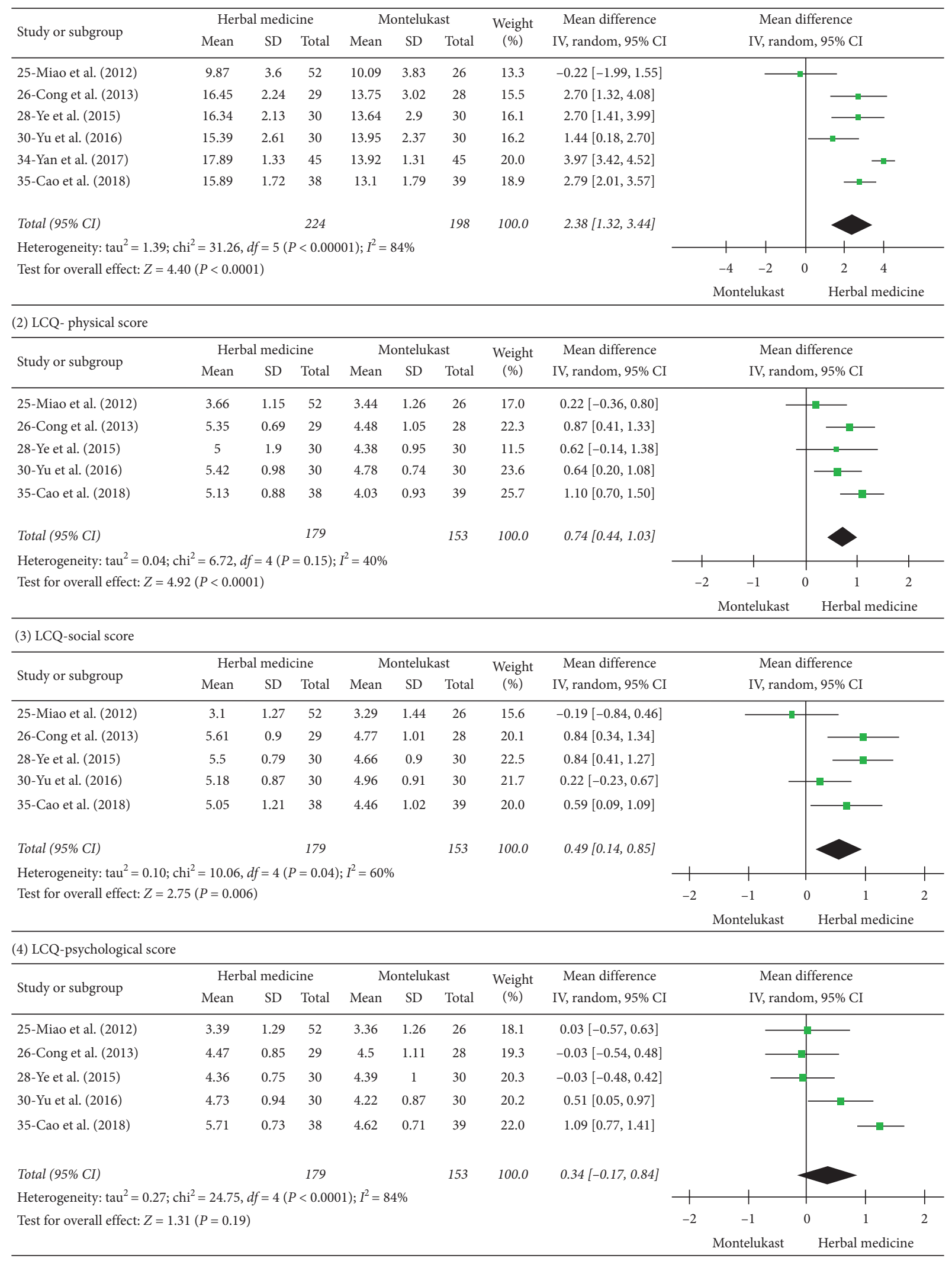

(a)

FIgURE 2: Continued. 


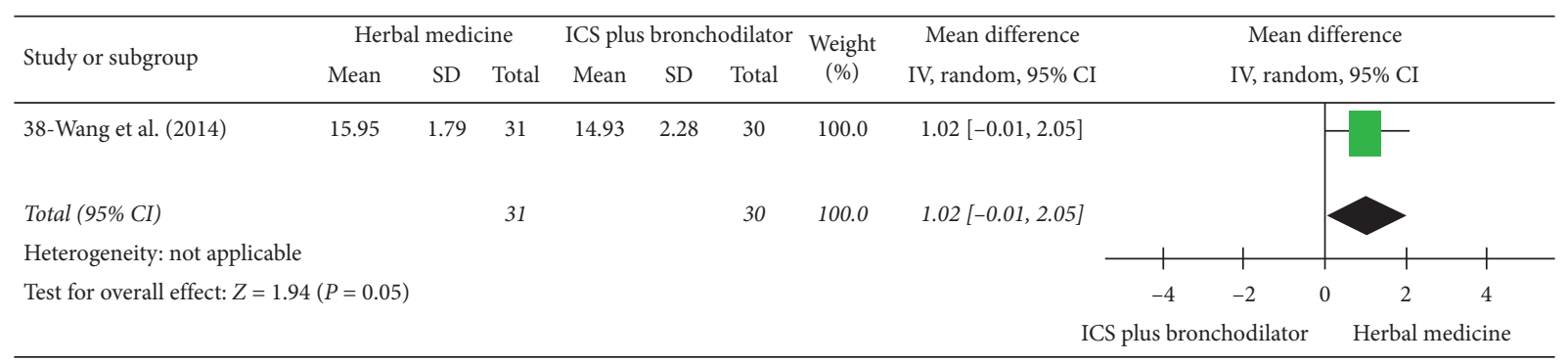

(b)

Figure 2: Forrest plot of Leicester Cough Questionnaire (LCQ). (a) Herbal medicine versus montelukast. (1) LCQ-total scores. (2) LCQphysical score. (3) LCQ-social score. (4) LCQ-psychological score. (b) Herbal medicine versus ICS plus bronchodilator.

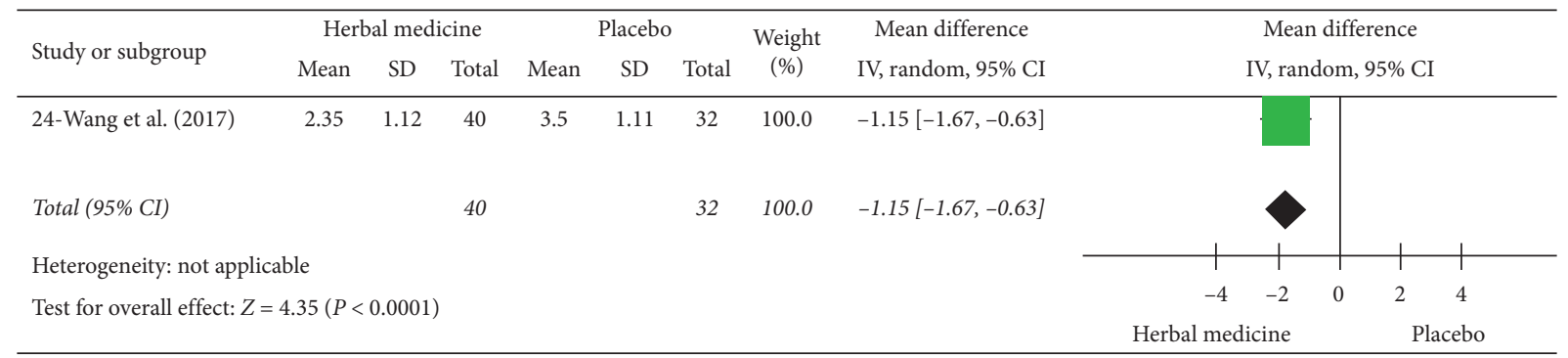

(a)

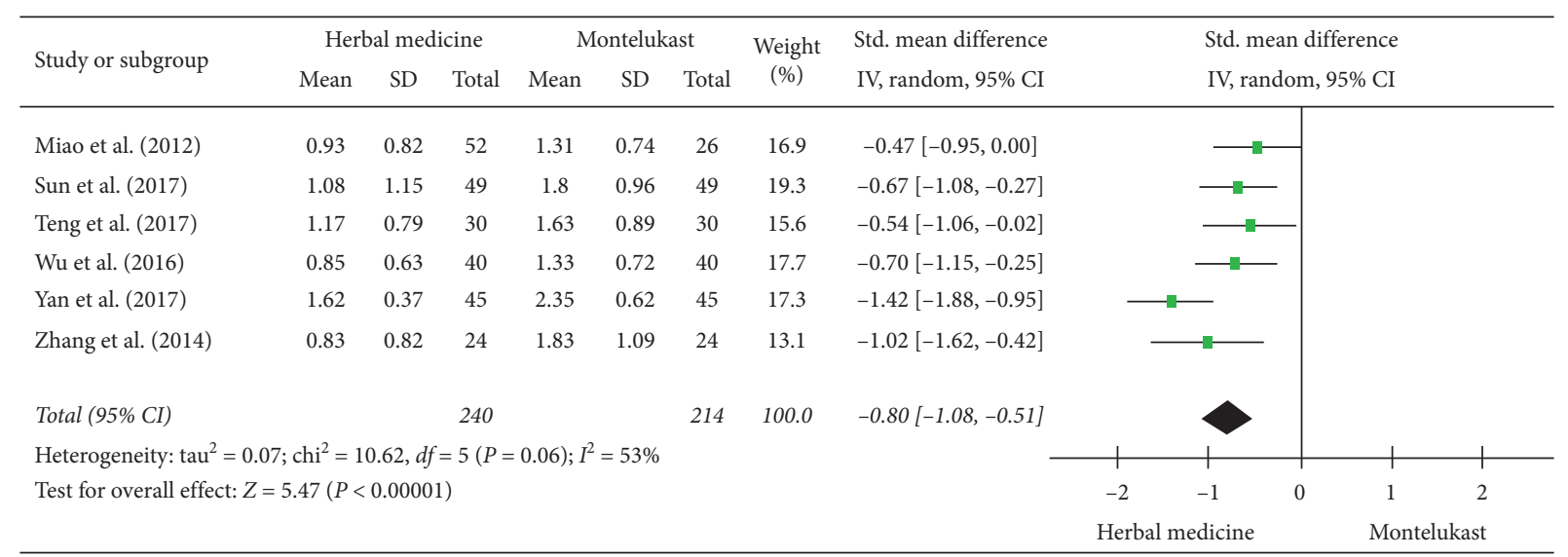

(b)

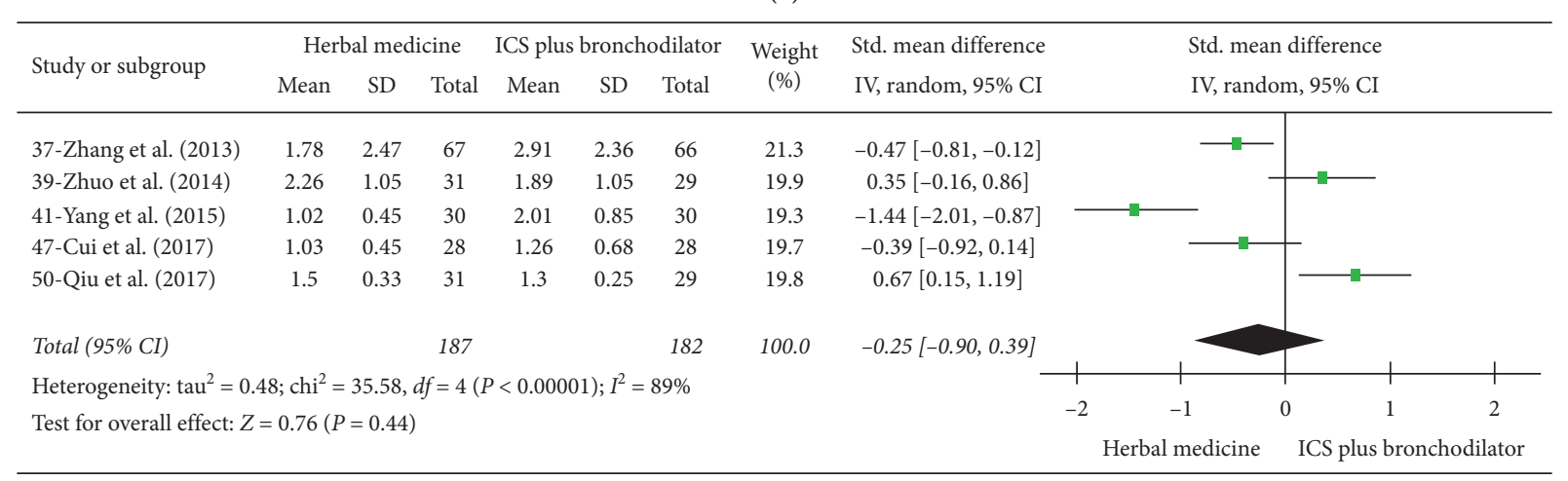

(c)

FIgURe 3: Forrest plot of cough symptom score (CSS). (a) Herbal medicine versus placebo. (b) Herbal medicine versus montelukast. (c) Herbal medicine versus ICS plus bronchodilator. 


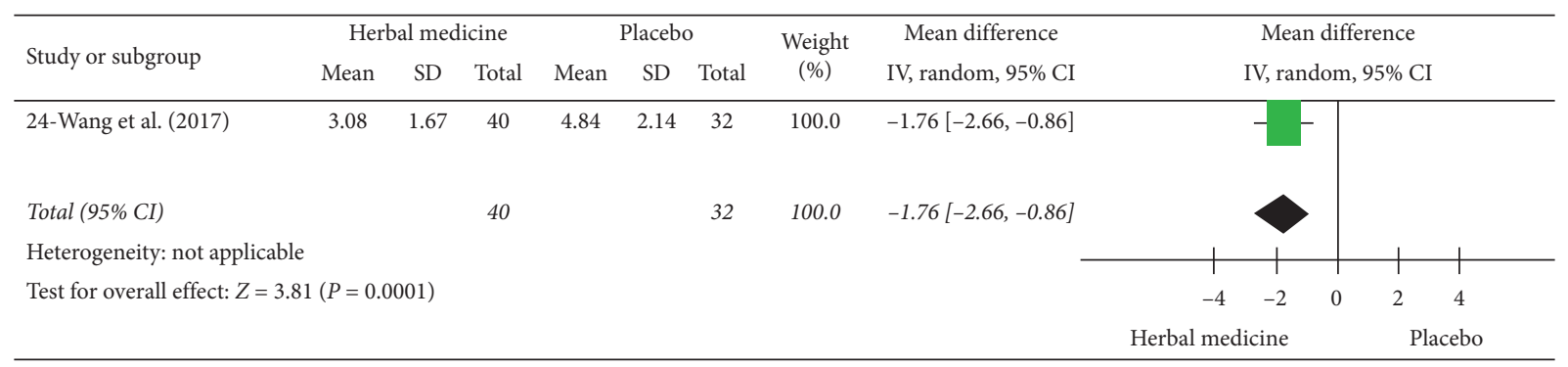

(a)

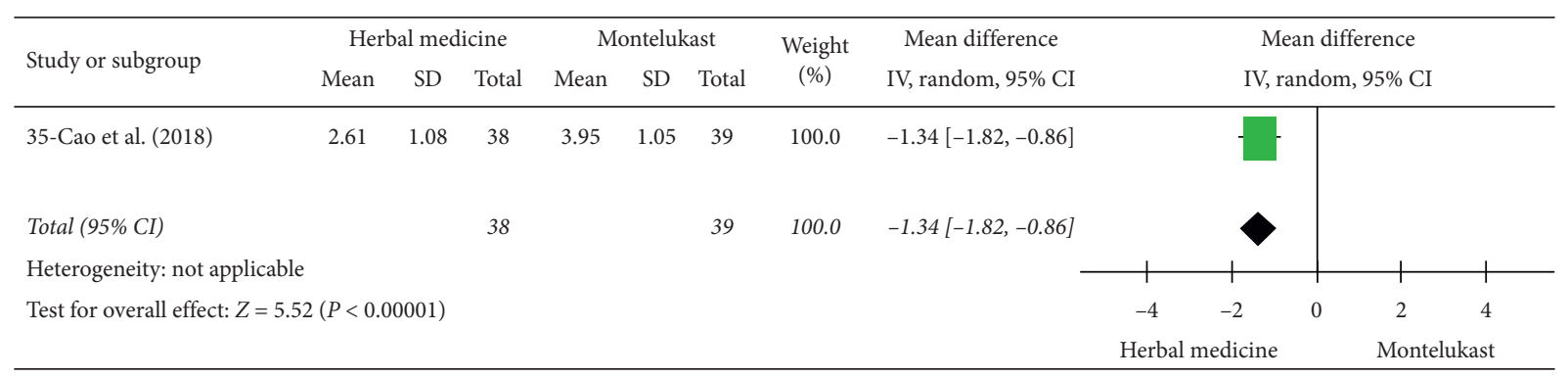

(b)

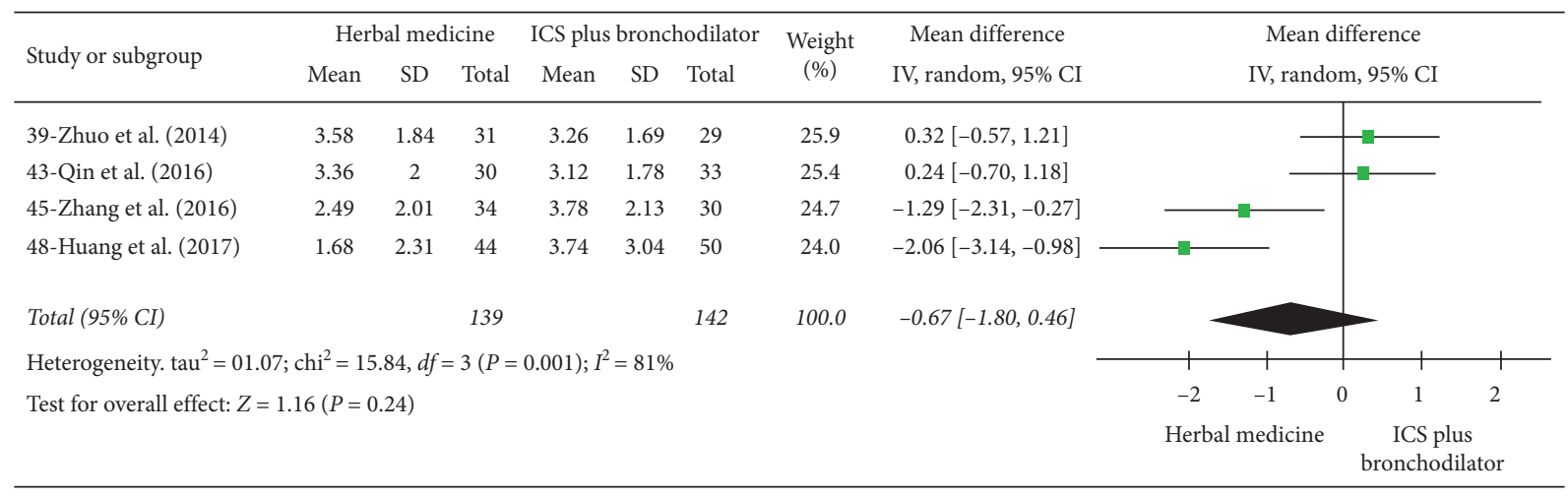

(c)

Figure 4: Forrest plot of visual analogue scale (VAS). (a) Herbal medicine versus placebo. (b) Herbal medicine versus montelukast. (c) Herbal medicine versus ICS plus bronchodilator.

controlled or markedly improved symptoms, and treatments were considered ineffective when people only reported a limited improvement or no symptom improvement. The overall result showed an improvement in the herbal medicine group compared to the montelukast group, and heterogeneity was moderate (RR $1.48 ; 95 \% \mathrm{CI}, 1.29$ to 1.71 ; $p<0.00001, I^{2}=44 \%$ ) (Figure S4).

\subsection{Results of Herbal Medicine versus ICS plus Bronchodilator}

3.4.1. Leicester Cough Questionnaire (LCQ). Low-quality evidence from one study of 61 participants showed that there was no difference between herbal medicine and ICS plus bronchodilator groups (MD 1.02 points; 95\% CI, -0.01 to 2.05) (Figure 2 and Table S2) [37].

3.4.2. Cough Symptom Score (CSS). Low-quality evidence from five studies with 369 participants assessed CSS (Table S2) [36, 38, 40, 46, 49]. The overall result indicated that participants receiving herbal medicine were not better than those receiving ICS plus bronchodilator, and heterogeneity was substantial (SMD -0.25 points; $95 \% \mathrm{CI},-0.90$ to $0.39 ; p=0.44, I^{2}=89 \%$ ) (Figure 3 ).

3.4.3. Visual Analogue Scale (VAS). Cough VAS was available in four studies which were judged as very lowquality evidence (281 participants) (Table S2) [38, 42, 44, 47]. The mean change in cough VAS was not statistically different between herbal medicine and ICS plus bronchodilator groups, and heterogeneity was considerable (MD -0.67 points; $95 \% \mathrm{CI},-1.80$ to $0.46 ; p=0.24, I^{2}=81 \%$ ) (Figure 4 ).

3.4.4. Forced Expiratory Volume in One Second (FEV1). ICS plus bronchodilator was used as a comparator in four RCTs with low-quality evidence $(n=287)$ evaluating $\mathrm{FEV}_{1}$ percent (Table S2) and one RCT $(n=118)$ evaluating $\mathrm{FEV}_{1}$ liters $[41,43,45,48] . \mathrm{FEV}_{1} \%$ increased in participants receiving herbal medicine compared to ICS plus 
bronchodilator, and heterogeneity might not be important (MD 3.83\%; 95\% CI, 1.55 to $6.10 ; p=0.001, I^{2}=36 \%$ ), but herbal medicine was not superior to ICS plus bronchodilator in terms of $\mathrm{FEV}_{1}(L)$ in one study (MD $-0.01 \mathrm{~L}$; $95 \% \mathrm{CI}$, -0.12 to 0.10 ) (Figure S3).

3.4.5. Effective Rate. Low-quality evidence from seven studies with 531 participants evaluated effective rate (Table S2) [35, 39-42, 46, 47]. The overall result showed that participants receiving herbal medicine were not better than those receiving ICS plus bronchodilator, and heterogeneity was substantial (RR $1.21 ; 95 \% \mathrm{CI}, 1.00$ to $1.47 ; p=0.05$, $\left.I^{2}=55 \%\right)$. Subgroup analysis was conducted on studies that had a duration of treatment less than four weeks or greater than four weeks. There was no significant advantage with a short treatment duration or longer treatment duration (RR 1.30; $95 \% \mathrm{CI}, 0.82$ to $2.08 ; p=0.27, I^{2}=70 \%$ and RR 1.14 ; $95 \% \mathrm{CI}, 0.95$ to $1.37 ; p=0.15, I^{2}=36 \%$, respectively) (Figure S4).

3.5. Results of Herbal Medicine versus Placebo. Two studies compared herbal medicine with placebo $[22,23]$. Moderatequality evidence from one study indicated that herbal medicine was superior to placebo and decreased the CSS (MD -1.15 points; $95 \% \mathrm{CI},-1.67$ to -0.63 ) and VAS (MD -1.76 points; $95 \% \mathrm{CI},-2.66$ to -0.86 ) (Figures 3 and 4 and Table S3) [23]. Participants receiving herbal medicine had an improved effective rate compared to participants taking placebo (RR 2.86; 95\% CI, 1.42 to 5.74) (Figure S4 and Table S3). Moderate-quality evidence from one study showed that immune function in terms of CD4+/CD8+ ratio in participants receiving herbal medicine was better than the placebo group ( $\mathrm{MD}-0.54 ; 95 \% \mathrm{CI},-0.73$ to -0.35$)$ (Figure S2 and Table S3) [22].

3.6. Sensitivity Analysis. In the sensitivity analysis for herbal medicine versus montelukast, four studies [27, 28, 30, 31] were excluded from the main analysis because they were identified as unclear risk of random sequence generation, and the overall results of LCQ and CSS did not change. One study [43] was excluded in the sensitivity analysis because of unclear risk of selection bias; the result indicated that herbal medicine was not superior to ICS plus bronchodilator in terms of $\mathrm{FEV}_{1}$ (\%) in three studies (MD 3.66; 95\% CI, -0.58 to $7.89, I^{2}=50 \%$ ) (Figure S5) $[41,45,48]$.

3.7. Safety and Adverse Events. Out of the 28 studies, 14
mentioned $[22,26,28,29,31,34-38,42,43,45,46]$. In one study that compared herbal medicine with placebo, there were no adverse events reported [22]. Five studies comparing herbal medicine with montelukast reported eight adverse events $[26,28,29,31,34]$. Constipation (2 cases) and diarrhea (2 cases) were reported in participants taking herbal medicine, while nausea ( 2 cases) and diarrhea ( 2 cases) were reported in the montelukast group. The incidence of any adverse event from eight studies was significantly higher in the participants receiving ICS plus bronchodilator than in those receiving herbal medicine (OR $0.23 ; 95 \% \mathrm{CI}, 0.07$ to 0.75 ; $p=0.01, I^{2}=11 \%$ ) (Figure 5) [35-38, 42, 43, 45, 46]. Only three events were reported in the herbal medicine group, including abdominal distension (2 cases) and nausea (1 case), but 22 adverse events were reported in participants taking ICS plus bronchodilator. Events included pharyngeal discomfort (11 cases), hoarseness of voice (8 cases), nausea (1 case), palpitation (1 case), and tremor (1 case).

\section{Discussion}

The systematic review included 28 RCTs involving 2,079 adult patients with CVA. Compared with placebo, herbal medicine reduced cough intensity and improved immune function, with a moderate level of certainty. Moderate- to low-quality evidence suggested that herbal medicine may also be associated with reduced cough symptoms and improved quality of life and $\mathrm{FEV}_{1}$ when compared with montelukast. However, herbal medicine was not superior to ICS plus bronchodilator, with a very low to a low level of certainty. The incidence and severity of adverse events were similar between montelukast and herbal medicine. Compared with ICS plus bronchodilator, relatively few adverse events from herbal medicine were reported, and ICS plus bronchodilator was associated with increased pharyngeal discomfort and hoarseness of voice. The meta-analysis provides an up-to-date analysis of herbal medicine for the treatment of adult CVA.

Cough variant asthma (CVA) features include airway hyperresponsiveness, atopy, and airway remodeling, as well as eosinophilic airway inflammation [51]. The efficacy of montelukast in treating cough due to asthma is related to its inhibition of inflammatory mediators such as leukotrienes [52]. The importance of leukotriene receptor antagonist was demonstrated in several guidelines, and systemic leukotriene antagonists such as montelukast in CVA have greater efficacy than classical asthma [53]. In the ACCP evidence-based guidelines, the leukotriene receptor antagonist is applied in a subgroup of patients whose cough had been refractory to therapy with inhaled steroids (quality of evidence, low; grade of recommendation, B) [12]. The ERS guidelines suggest a short-term antileukotriene trial (2-4 weeks) in adult patients with asthmatic cough (conditional recommendation, lowquality evidence) [54]. The leukotriene receptor antagonist is also recommended by Chinese guidelines because it reduces cough symptoms and airway inflammation (grade of recommendation, B) [13].

We found an improved association with the benefits of oral herbal medicine (for 2-4 weeks) in reducing cough in terms of physical and social scores on the LCQ over montelukast. The results also showed potential benefits of herbal medicine in reducing subjective cough frequency and severity scores, as well as improving lung function. Moreover, in terms of safety, herbal medicine was similar to montelukast. Herbal medicine as a monotherapy is more effective with regard to clinical outcomes than montelukast, although this finding was based on moderate- to low-quality evidence. 


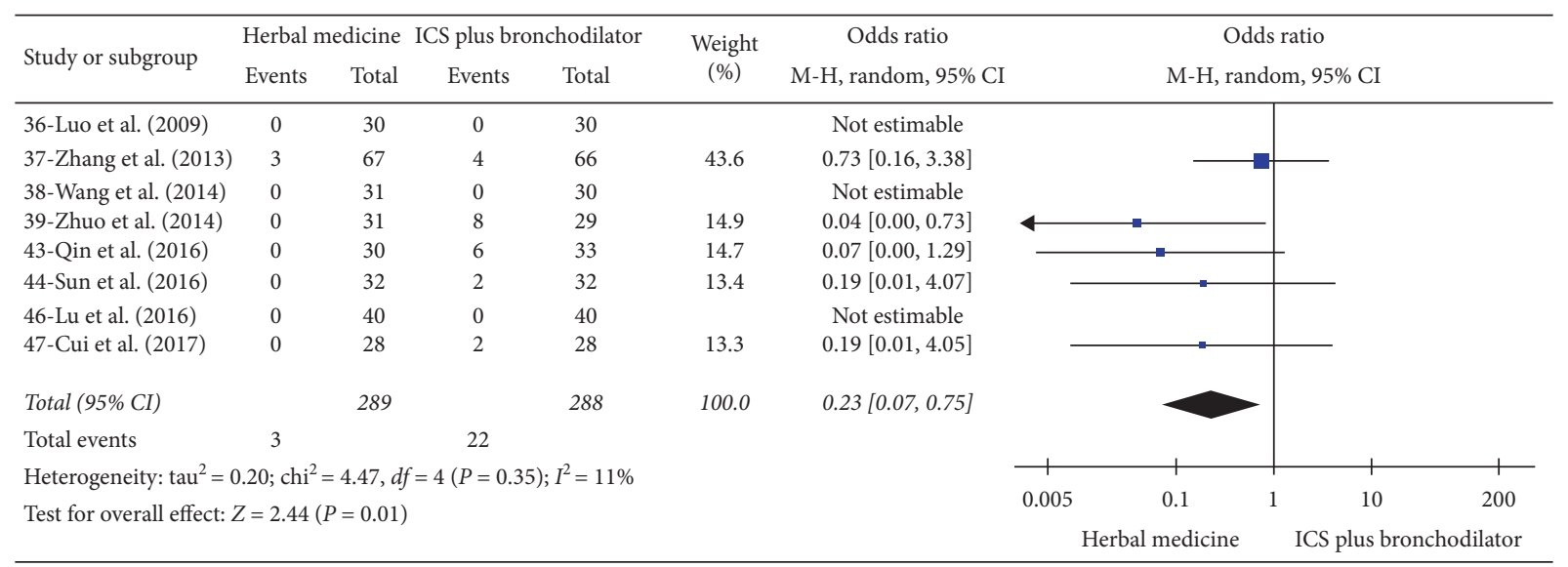

FIGURE 5: Incidence of adverse events in herbal medicine versus ICS plus bronchodilator.

The positive results from two double-blind, placebocontrol studies also suggest the potential efficacy of herbal medicine in improving subjective cough scores and regulating immune function.

Almost all clinical practice guidelines recommend ICS plus bronchodilator as the standard treatment for CVA $[12,13,54,55]$. Treatment is expected to be effective in the short term (within 2-4 weeks) [12, 54, 55], except Chinese guidelines that suggested the duration of treatment should be more than eight weeks and some patients require longterm treatment [13]. The standardized treatment usually has a good effect, especially corticosteroids because eosinophils are implicated in CVA and corticosteroids reduce eosinophilic inflammation [51]. However, there are some patients with CVA that are refractory to treatment with ICS plus bronchodilator. Furthermore, a reduced association with a clinical benefit over time might lead to the prescription of long-term or higher corticosteroid doses and consequently more risk of adverse events.

Our review revealed that herbal medicine did not significantly improve subjective cough outcomes and quality of life compared to ICS plus bronchodilator, but the risk of adverse events due to ICS plus bronchodilator was seven times higher than herbal medicine. Because of the possibly better long-term safety profile of herbal medicine, it may be considered as an add-on treatment alternative in CVA in suboptimally controlled patients taking ICS plus bronchodilator. Where medical decision-making is concerned, we should not only pay attention to the availability of cough control therapies and the patient's response in terms of symptom relief but also consider the side effects.

Herbal medicine has therapeutic effects largely attributable to its active compounds. Experimental evidence including in vitro and in vivo studies helps to explain the possible mechanisms of action of the herbs and how they may improve the signs and symptoms of chronic cough. There are still a limited number of CVA models, and the majority of experimental studies focus on surrogate outcomes such as anti-inflammation, antioxidation, antiallergy, or immunomodulation [56-58]. Many antitussive herbs including Gan cao (Glycyrrhiza spp.) [59], Ma huang (Ephedra sinica Stapf) [60], and Chan tui (Cryptotympana pustulata Fabricius) [61] are likely to have antispasmodic or bronchodilator actions, although these effects are yet to be explored in experimental models. The research indicates that herbal medicine has important actions that interact and modulate the key pathophysiological mechanisms associated with CVA. Potentially, there are additional benefits of the herbs on associated pathogenesis that have not been fully researched in animal models of cough but show promise.

4.1. Limitations. This review had several limitations. First, there was considerable heterogeneity among the included trials in some outcome measures. Second, the results could not demonstrate the differences between the various leukotriene receptor antagonists and herbal medicines, because the studies did not include other antileukotriene drugs except for montelukast, and the efficacy and safety profiles may be not the same. Third, the evaluation of CVA is mainly based on subjective outcomes, but most of the included studies were openlabel trials; therefore, bias cannot be ruled out. Finally, the methodological quality was low to moderate, and the results of this meta-analysis should be interpreted with caution because none of the included studies were free from bias.

4.2. Suggestions. What is the role of herbal medicine in adult patients with CVA? Current guidelines lack specific recommendations as to how and when herbal medicine should be used for patients with CVA. The results of our review suggest that herbal medicine could be considered in the following situations: (1) in patients who did not respond or had a suboptimal response to ICS plus bronchodilator treatments, (2) in patients who did not respond or had a suboptimal response to montelukast, and (3) in patients who have a strong preference to take herbal medicine.

\section{Conclusions}

In this meta-analysis of RCTs of adult patients with CVA, herbal medicine was superior to placebo and montelukast but not ICS plus bronchodilator, based on very low- to moderate-level evidence. Herbal medicine was safe and associated with a lower incidence of adverse events 
compared with ICS plus bronchodilator. These findings indicate that people with chronic cough may benefit from herbal medicine. As new research emerges, specific recommendations for their use should be updated, especially in countries where herbal medicine is a significant part of the health care system such as in China.

$\begin{array}{ll}\text { Abbreviations } \\ \text { CSS: } & \text { Cough symptom score } \\ \text { CVA: } & \text { Cough variant asthma } \\ \text { FEV1: } & \text { Forced expiratory volume in one second } \\ \text { GRADE: } & \text { Grading of Recommendations Assessment, } \\ & \text { Development, and Evaluation } \\ \text { ICS: } & \text { Inhaled corticosteroid } \\ \text { LCQ: } & \text { Leicester Cough Questionnaire } \\ \text { MD: } & \text { Mean difference } \\ \text { MCID: } & \text { Minimal clinically important difference } \\ \text { OR: } & \text { Odds ratios } \\ \text { RCTs: } & \text { Randomized controlled trials } \\ \text { RR: } & \text { Risk ratio } \\ \text { SMD: } & \text { Standardized mean difference } \\ \text { VAS: } & \text { Visual analogue scale. }\end{array}$

\section{Data Availability}

All the data of this review are available from the public, open-access electronic databases.

\section{Conflicts of Interest}

The authors declare that they have no conflicts of interest.

\section{Authors' Contributions}

All authors contributed substantially to the design, interpretation of the data, statistical analysis, drafting the manuscript, and approving the submission. The first two authors contributed equally to the study.

\section{Acknowledgments}

The authors thank the contribution of the China-Australia International Research Centre for Chinese Medicine to this work. This study was supported by the Construction Project of the TCM Clinical Research Base of the State Administration of TCM (Grant no. JDZX2015199), the Science and Technology Research Project of Guangdong Provincial Hospital of Chinese Medicine (Grant no. YN2014PJ02), the Natural Science Foundation of Guangdong Province (Grant no. 2017A030313724), Guangdong Provincial Science and Technology Innovation Strategy Special Fund (GuangdongHong Kong-Macau Joint Lab, Grant no. 2020B1212030006), and Science and Technology Planning Project of Guangzhou (Grant no. 202002020042).

\section{Supplementary Materials}

Figure S1: risk of bias graph; Figure S2: forest plot of CD4+/ $\mathrm{CD} 8+$ ratio; Figure S3: forest plot of forced expiratory volume in one second (FEV1); Figure S4: forest plot of effective rate; Figure S5: sensitivity analyses of FEV1 (\%) in herbal medicine versus ICS plus bronchodilator; Table S1: GRADE evaluation of herbal medicine versus montelukast; Table S2: GRADE evaluation of herbal medicine versus ICS plus bronchodilators; Table S3: GRADE evaluation of Chinese herbal medicine versus placebo. (Supplementary Materials)

\section{References}

[1] M. D. Lougheed, S. E. Turcotte, and T. Fisher, "Cough variant asthma: lessons learned from deep inspirations," Lung, vol. 190, no. 1, pp. 17-22, 2012.

[2] R. S. Irwin and J. M. Madison, "The persistently troublesome cough," American Journal of Respiratory and Critical Care Medicine, vol. 165, no. 11, pp. 1469-1474, 2002.

[3] R. S. Irwin, C. L. French, A. B. Chang, and K. W Altman, "Classification of cough as a symptom in adults and management algorithms: CHEST guideline and expert panel report," Chest, vol. 153, no. 1, pp. 196-209, 2018.

[4] K. Lai, J. Pan, R. Chen, B. Liu, W. Luo, and N. Zhong, "Epidemiology of cough in relation to China," Cough, vol. 9, no. 1, p. 18, 2013.

[5] A. Niimi, H. Ohbayashi, H. Sagara et al., "Cough variant and cough-predominant asthma are major causes of persistent cough: a multicenter study in Japan," Journal of Asthma, vol. 50, no. 9, pp. 932-937, 2013.

[6] P. G. Gibson and A. E. Vertigan, "Management of chronic refractory cough,” BMJ, vol. 351, p. h5590, 2015.

[7] A. H. Morice, G. A. Fontana, A. R. Sovijarvi et al., "The diagnosis and management of chronic cough," European Respiratory Journal, vol. 24, no. 3, pp. 481-492, 2004.

[8] A. Emeryk, R. Klink, T. Mciver, and P. Dalvi, “A 12-week openlabel, randomized, controlled trial and 24-week extension to assess the efficacy and safety of fluticasone propionate/formoterol in children with asthma," Therapeutic Advances in Respiratory Disease, vol. 10, no. 4, pp. 324-337, 2016.

[9] H. H. Raissy, H. W. Kelly, M. Harkins, and S. J. Szefler, "Inhaled corticosteroids in lung diseases," American Journal of Respiratory and Critical Care Medicine, vol. 187, no. 8, pp. 798-803, 2013.

[10] K. Peltzer and S. Pengpid, "The use of herbal medicines among chronic disease patients in Thailand: a cross-sectional survey," Journal of Multidisciplinary Healthcare, vol. 12, no. 22, pp. 573-582, 2019.

[11] M. Tulunay, C. Aypak, H. Yikilkan, and S. Gorpelioglu, "Herbal medicine use among Turkish patients with chronic diseases," Journal of Intercultural Ethnopharmacology, vol. 4, no. 3, pp. 217-220, 2015.

[12] American College of Chest Physicians, "Diagnosis and management of cough: ACCP evidence-based clinical practice guidelines," Chest, vol. 129, pp. 1S-292S, 2006.

[13] Asthma Workgroup of the Chinese Society of Respiratory Diseases, "The chinese national guidelines on diagnosis and management of cough," Chinese Medical Journal, vol. 32, no. 6, pp. 407-413, 2009.

[14] J. P. T. Higgins and S. Green, Cochrane Handbook for Systematic Reviews of Interventions, Cochrane, 2016, http:// training.cochrane.org/handbook.

[15] D. Moher, A. Liberati, J. Tetzlaff, and D. G. Altman, "Preferred reporting items for systematic reviews and meta-analyses: the 
PRISMA statement," International Journal of Surgery, vol. 8, no. 5, pp. 336-341, 2010.

[16] S. S. Birring, B. Prudon, A. J. Carr et al., "Development of a symptom specific health status measure for patients with chronic cough: Leicester Cough Questionnaire (LCQ)," Thorax, vol. 58, no. 4, pp. 339-343, 2003.

[17] Asthma Workgroup of the Chinese Society of Respiratory Diseases, "The chinese national guidelines on diagnosis and management of cough," Chinese Medical Journal, vol. 124, pp. 3207-3219, 2011.

[18] J. Y. Hsu, R. A. Stone, R. B. Logan-Sinclair, M. Worsdell, C. M. Busst, and K. F. Chung, "Coughing frequency in patients with persistent cough: assessment using a 24 hour ambulatory recorder," European Respiratory Journal, vol. 7, no. 7, pp. 1246-1253, 1994.

[19] Ministry of Health of the PRC, Clinical Guidelines of New Drugs of Traditional Chinese Medicine, People's Health Press, Beijing, China, 2002.

[20] State Administration of Traditional Chinese Medicine, Criteria of Diagnosis and Therapeutic Effect of Diseases and Syndromes in Traditional Chinese Medicine, Nanjing University Press, Nanjing, China, 1995.

[21] G. H. Guyatt, A. D. Oxman, G. E. Vist et al., "GRADE: an emerging consensus on rating quality of evidence and strength of recommendations," BMJ, vol. 336, no. 7650, pp. 924-926, 2008.

[22] Y. Zhang, Z. D. Zhang, and X. Zhang, "Effect of Yupingfeng Powder on immune function in patients with cough variant asthma in stable stage," Guangzhou University of Chinese Medicine, vol. 26, no. 6, pp. 517-519, 2009.

[23] Q. Wang, "A study of effect rate of expelling wind and releasing lung formula to cure CVA and its relating effect to airway neurogenic inflammation," Beijing University of Chinese Medicine, 2017.

[24] Q. Q. Miao, "Clinical study on cough variant asthma based on Zhisou San," Master thesis, Beijing University of Chinese Medicine, Beijing, China, 2012.

[25] X. D. Cong, "Effect of wen run xin jin formula on cough variant asthma with wind cold attacking lungs syndrome," Master thesis, Beijing University of Chinese Medicine, Beijing, China, 2013.

[26] F. Zhang, "Clinical Effect of Hua gai san modified decoction in the treatment of Cough Variant Asthma with severe wind attacking lungs syndrome," Master thesis, Chengdu University of Traditional Chinese Medicine, Chengdu, China, 2014.

[27] Y. Ye, H. W. Jin, and Z. R. Li, "Effect of strengthening spleen and clearing lungs on quality of life in patients with cough variant asthma due to phlegm-dampness obstructing the Lungs," Jilin-Medical-University, vol. 36, no. 10, p. 2096, 2015.

[28] Z. H. Wu, "Clinical observation of applying nourishing zongqi method in the treatment of cough variant asthma," Journal of Sichuan Traditional Chinese Medicine, vol. 34, no. 08, pp. 179-180, 2016.

[29] J. Yu, The Clinical Research On Qufengxuanfei Method in the Treatment of Cough Variant Asthma with Wing Phlegm Obstructing Lung Syndrome, Nanjing University of Chinese Medicine, Nanjing, China, 2016.

[30] N. S. Kang, W. M. Ma, X. T. Chen et al., "Clinical effect of Chanyi formula in the treatment of cough variant asthma," Journal of Integrated Traditional and Western Medicine, vol. 27, no. 02, pp. 123-125, 2017.
[31] Y. H. Sun, Y. X. Wang, and X. M. Ma, "Clinical study on Suhuang Zhike capsules for cough variant asthma," Trace Element Research, vol. 34, no. 06, pp. 7-9, 2017.

[32] C. Teng, "Clinical observation of Zhike Pingchuan formula on cough variant asthma with severe wing attacking lungs and its effect on EOS and IL-10," Master thesis, Heilongjiang University of Chinese Medicine, Heilongjiang, China, 2017.

[33] X. Yan and Q. Wang, "Clinical observation on yiqi qufeng formula for cough variant asthma," Chinese Journal Of Information on TCM, vol. 24, no. 11, pp. 30-33, 2017.

[34] F. Z. Cao, "Clinical observation on modified maxing Erchen formula in the treatment of elderly cough variant asthma," Master thesis, Yunnan University of Traditional Chinese Medicine, Kunming, China, 2018.

[35] J. H. Luo, "Clinical observation of Keping formula in the treatment of cough variant asthma with severe wind attacking lungs," Master thesis, Fujian Medical University, Fujian, China, 2009.

[36] G. C. Zhang, Z. Y. Chou, Y. P. Cai et al., "Clinical study on Guben Zhike Granule in the treatment of cough variant asthma," Chinese Journal of Integrative Medicine, vol. 20, no. 12, pp. 9-11, 2013.

[37] Q. L. Wang, "Clinical observation of supplemented Gallbladder-warming formula in the treatment of cough variant asthma with wind and phlegm-heat attacking lungs," Master thesis, Fujian Medical University, Fujian, China, 2017.

[38] Z. Y. Zhuo, "Clinical observation of budesonide formoterol powder inhalation combined with Suhuangzhike capsules in the treatment of cough variant asthma," Cerebral Vascular Disease, vol. 22, no. 12, pp. 91-92, 2014.

[39] D. Y. Xin, "Clinical observation of Jiuxian san in the treatment of cough variant asthma," Traditional Chinese Medicine, vol. 32, no. 01, pp. 97-99, 2015.

[40] Y. Y. Yang and X. H. Chen, "Clinical observation on Tiaogan Sufei formula for cough variant asthma," Shanghai University of Traditional Chinese Medicine, vol. 29, no. 01, pp. 30-32, 2015.

[41] H. X. Zhao and H. J. Feng, "Clinical observation of tonifying Qi and Spleen combined with salmeterol/fluticasone in the treatment of cough variant asthma," Chinese Journal of Integrative Medicine, vol. 17, no. 03, pp. 282-283, 2015.

[42] W. Qing, "Effect of modified Chaihu Zhiju formula on IL-12 and total IgE in patients with cough variant asthma," Master thesis, Yunnan University of Traditional Chinese Medicine, Kunming, China, 2016.

[43] H. C. Sun, "Clinical study on yangyin qufeng method for cough variant asthma," Master thesis, Nanjing University of Chinese Medicine, Nanjing, China, 2016.

[44] Y. P. Zhang, "Clinical study on combination of Chinese and western medicine for cough variant asthma with windphlegm Syndrome," Master thesis, Nanjing University of Chinese Medicine, Nanjing, China, 2016.

[45] S. X. Lu, B. M. Li, L. H. Yin et al., "Clinical study on Shufeng Zhisou formula in the treatment of cough variant asthma," Chinese Journal of Integrative Medicine, vol. 23, no. 12, pp. 30-33, 2016.

[46] Y. Cui, "Shuchen Wang academic thoughts and clinical experience and kidney functions of treatment of cough variant asthma clinical studies," Doctor thesis, Chinese Academy of Medical Sciences, Beijing, China, 2017.

[47] Z. K. Huang, X. T. Wu, X. A. Zhao et al., "Clinical observation on Qufeng Zhike formula in the Treatment of 44 Cases of patients with cough variant asthma," Guangming Journal Chinese Medicine, vol. 32, no. 19, pp. 2780-2782, 2017. 
[48] S. X. Yin, L. H. Yin, B. M. Li et al., "Clinical observation on Tiaozhong Yifei formula combined with western medicine in the stable stage of cough variant asthma," Chinese Journal of Integrative Medicine, vol. 24, no. 7, pp. 36-39, 2017.

[49] R. Qiu, C. W. Xiao, and F. Q. Wen, "Efficacy observation of budesonide/formoterol combined with Suhuang Zhike capsules on cough variant asthma," Journal of Clinical Pulmonary Medicine, vol. 22, no. 2, pp. 304-306, 2017.

[50] K. F. Chung and J. G. Widdicombe, "Pharmacology and therapeutics of cough. Preface," Handbook of Experimental Pharmacology, vol. 187, 2009.

[51] A. Niimi, "Cough and asthma," Current Respiratory Medicine Reviews, vol. 7, no. 1, pp. 47-54, 2011.

[52] P. V. Dicpinigaitis, J. B. Dobkin, and J. Reichel, "Antitussive effect of the leukotriene receptor antagonist zafirlukast in subjects with cough-variant asthma *," Journal of Asthma, vol. 39, no. 4, pp. 291-297, 2002.

[53] M. Takemura, A. Niimi, H. Matsumoto et al., "Clinical, physiological and anti-inflammatory effect of montelukast in patients with cough variant asthma," Respiration, vol. 83, no. 4, pp. 308-315, 2012.

[54] A. H. Morice, E. Millqvist, K. Bieksiene et al., "ERS guidelines on the diagnosis and treatment of chronic cough in adults and children," The European Respiratory Journal, vol. 9, no. 12, Article ID 1901136, 2019.

[55] P. G. Gibson, A. B. Chang, N. J. Glasgow et al., "CICADA: cough in children and adults: diagnosis and assessment. Australian cough guidelines summary statement," Medical Journal of Australia, vol. 192, no. 5, pp. 265-271, 2010.

[56] J. P. Joad, P. A. Munch, J. M. Bric et al., "Passive smoke effects on cough and airways in young Guinea pigs," American Journal of Respiratory and Critical Care Medicine, vol. 169, no. 4, pp. 499-504, 2004.

[57] D. J. Prockop and J. Youn Oh, "Mesenchymal stem/stromal cells (MSCs): role as guardians of inflammation," Molecular Therapy, vol. 20, no. 1, pp. 14-20, 2012.

[58] G. R. Saraswathy, R. Sathiya, J. Anbu et al., "Antitussive medicinal herbs- an update review," International Journal of Pharmaceutical Sciences and Drug Research, vol. 6, no. 1, pp. 12-19, 2004.

[59] Q. Zhang and M. Ye, "Chemical analysis of the Chinese herbal medicine Gan-Cao (licorice)," Journal of Chromatography A, vol. 1216, no. 11, pp. 1954-1969, 2009.

[60] C.-H. Ma, Z.-Q. Ma, Q. Fu, and S.-P. Ma, "Ma Huang Tang ameliorates asthma though modulation of Th1/Th2 cytokines and inhibition of Th17 cells in ovalbumin-sensitized mice," Chinese Journal of Natural Medicines, vol. 12, no. 5, pp. 361-366, 2014.

[61] H. Jiang, S. Yang, and H. Chen, "Screening of effective parts of cicada slough for relieving cough, phlegm and asthma," Chinese Journal of Integrative Medicine, vol. 19, no. 1, pp. 56-59, 2017. 